\title{
NONLOCAL MULTISCALE INTERACTIONS IN BRAIN NEURODEGENERATIVE PROTEIN DYNAMICS AND COUPLED PROTEOPATHIC PROCESSES
}

\author{
Swadesh Pal ${ }^{a}$ and Roderick Melnik ${ }^{*, a, b}$ \\ ${ }^{a}$ M3AI Laboratory, MS2Discovery Interdisciplinary Research Institute \\ Wilfrid Laurier University, Waterloo, ON, Canada N2L 3C5 \\ e-mail: palkkeswadesh@gmail.com \\ ${ }^{b}$ BCAM - Basque Center for Applied Mathematics, E-48009, Bilbao, Spain \\ e-mail: rmelnik@wlu.ca - Web page: http://m3ai.wlu.ca/
}

Key words: Nonlocal Interactions, Coupled Problems, Multiscale Model, Neurodegenerative Disorders, Brain Connectome, Alzheimer Disease, Network-based Computations

\begin{abstract}
We take a heterodimer multiscale reaction-diffusion model as a starting point and modify it to account for the nonlocality of the underlying processes. The resulting model is a coupled system of integro-differential equations. The effect of nonlocal interactions is quantified based on the comparisons of the newly developed model with its local counterpart. Our primary focus is given to the estimation of conversion times from healthy to toxic proteins. This is done based on the stability analysis of a simplified model, as well as on the fully coupled numerical implementation. Several clinically relevant patient proteopathies are highlighted. They include the primary tauopathy, as well as the secondary tauopathy where the sustained presence of toxic tau protein requires also to be accompanied by toxic amyloid-beta peptides. We demonstrate the dynamics of concentrations of toxic proteins under several clinically realistic scenarios. Finally, we carry out a detailed parametric study, providing further insight into the role of nonlinear effects controlled via the Holling type II functional response.
\end{abstract}

\section{INTRODUCTION}

In neurodegenerative diseases, a particular intervention based on advanced biomedical engineering is often hindered by our lack of understanding of coupled, concomitant proteinopathies [1]. While there is a continuing debate in the literature about the roles of the proteostasis of amyloid-beta peptides and the microtubule-associated tau protein, it is known that aging alters them both. The processes related to their changes are not independent, they are coupled [2]. Our better understanding of this and other types of 
couplings is critical to disease-specific diagnosis, prevention, and treatment of neurodegenerative diseases in general and Alzheimer's disease (AD) in particular [3, 4].

$\mathrm{AD}$ is the most common type of neurodegenerative disorder (ND) that increases degrees of dementia over time [5]. It has been reported that 5.5 million Americans in the U.S. and 44 million worldwide deal with this disease in all ranges of ages, while $10 \%$ of the population is suffering over 65 years of age [6]. According to the pathological report of $\mathrm{AD}$, one of the main reasons for the loss of nerves and synapses is the accumulation of extracellular amyloid plaques peptides and intracellular neurofibrillary tangles (NFTs) in the nervous system $[7,8]$. Generally, tau protein helps to stabilize microtubules within the axon, but when it forms NFTs, it acts as a seed for further misfolding and aggregation. The aggregation of tau proteins happens in the axon and then is transported into extracellular space [9]. Diffusion occurs in that space through secretion and then damage the host cell [10].

The accumulation of these proteins has been studied mathematically [11]. Multiple modes based on differential equations have been used for describing AD. However, a number of questions regarding this disease remain open and more refined models need to be developed to address them. Here, we use a reaction-diffusion equation with a modified heterodimer model in the reaction kinetics to describe the interaction between amyloid-

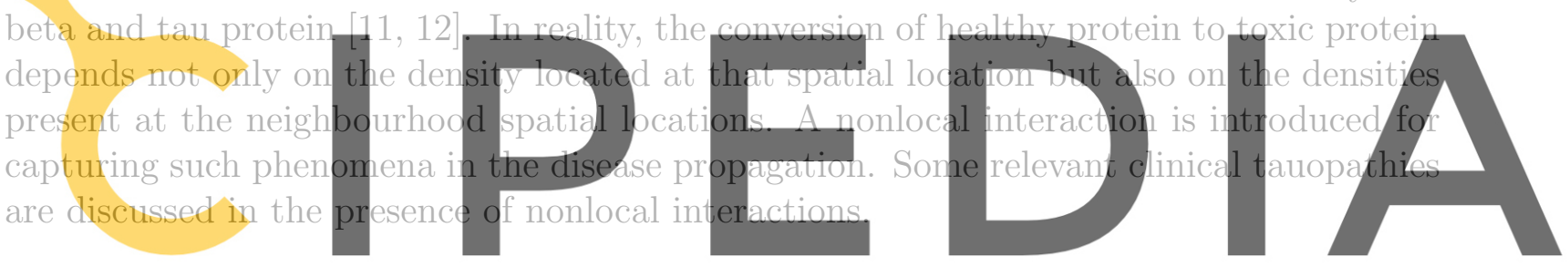

\section{MATHEMATICAL MODEL}

Register for free at https/dWww.scipedia.com to download the version without the watermark

We consider $\Omega \subset \mathbb{R}^{2}$ is a spatial domain. For $x \in \Omega$ and time $t \in \mathbb{R}^{+}$, we denote by $u=u(\mathbf{x}, t)$ and $v=v(\mathbf{x}, t)$, the concentrations of healthy $A \beta$ and $\tau P$ respectively. Similarly, we denote by $\widetilde{u}=\widetilde{u}(\mathbf{x}, t)$ and $\widetilde{v}=\widetilde{v}(\mathbf{x}, t)$, the concentrations of toxic $A \beta$ and $\tau P$ respectively. The evolution of concentrations of the four populations can then be given as follows [12]:

$$
\begin{aligned}
& \frac{\partial u}{\partial t}=\nabla \cdot\left(\mathbf{D}_{1} \nabla u\right)+a_{0}-a_{1} u-\frac{a_{2} u}{1+e_{1} u} \widetilde{u}, \\
& \frac{\partial \widetilde{u}}{\partial t}=\nabla \cdot\left(\widetilde{\mathbf{D}}_{1} \nabla \widetilde{u}\right)-\widetilde{a}_{1} \widetilde{u}+\frac{a_{2} u}{1+e_{1} u} \widetilde{u}, \\
& \frac{\partial v}{\partial t}=\nabla \cdot\left(\mathbf{D}_{2} \nabla v\right)+b_{0}-b_{1} v-\frac{b_{2} v}{1+e_{2} v} \widetilde{v}-b_{3} \widetilde{u} v \widetilde{v}, \\
& \frac{\partial \widetilde{v}}{\partial t}=\nabla \cdot\left(\widetilde{\mathbf{D}}_{2} \nabla \widetilde{v}\right)-\widetilde{b}_{1} \widetilde{v}+\frac{b_{2} v}{1+e_{2} v} \widetilde{v}+b_{3} \widetilde{u} v \widetilde{v},
\end{aligned}
$$


where the first two equations correspond to the usual heterodimer model for the healthy and toxic variants of the protein $u$ and the last two equations are the same for $v$. Here, $a_{0}$ and $b_{0}$ are the mean production rates of healthy proteins, $a_{1}, b_{1}, \widetilde{a}_{1}$ and $\widetilde{b}_{1}$ are the mean clearance rates of healthy and toxic proteins, and $a_{2}$ and $b_{2}$ represent the mean conversion rates of healthy proteins to toxic proteins. The parameter $b_{3}$ is the coupling between the two proteins $A \beta$ and $\tau P$. Here, $e_{1}$ and $e_{2}$ are the dimensionless parameters that describe the conversion time of the healthy protein to the toxic protein of $A \beta$ and $\tau P$, respectively. The diffusion tensors $\mathbf{D}_{1}, \widetilde{\mathbf{D}}_{1}, \mathbf{D}_{2}$ and $\widetilde{\mathbf{D}}_{2}$ characterize the spreading of each proteins. We assume all variables and initial conditions to be non-negative, and all the parameters to be strictly positive.

\subsection{Nonlocal model}

In model (1), the conversion of toxic protein from the healthy protein at a spatial point $\mathbf{x}$ depends only on the healthy proteins located at the same spatial point $\mathbf{x}$. In general, the converted toxic protein located at a spatial point $\mathbf{x}$ depends on the healthy proteins located in the neighbourhood of the spatial point $\mathbf{x}$. Under this assumption, we modify the model (1), inspired by the ideas presented in [13]:
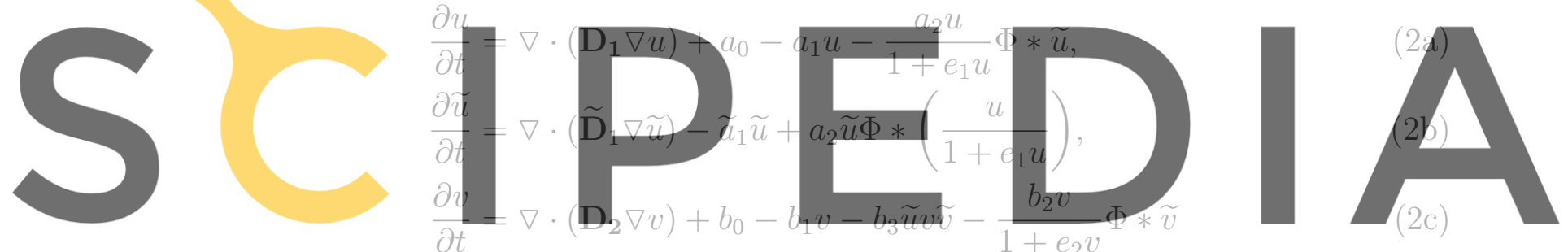

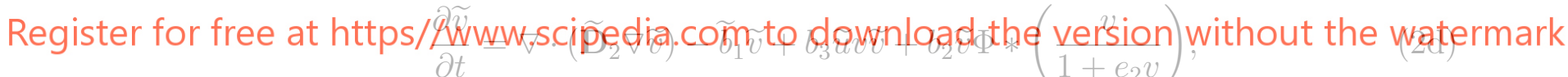

where the convolution $\Phi * \widetilde{u}$ is defined by $(\Phi * \widetilde{u})(\mathbf{x}, t)=\int_{\Omega} \Phi(\mathbf{x}-\mathbf{y}) \widetilde{u}(\mathbf{y}, t) d \mathbf{y}$ with $\int_{\Omega} \Phi(\mathbf{x}) d \mathbf{x}=1$. Similarly, we define the other convolutions. Here, $\Phi$ is the kernel functions that describe the conversion efficiencies between the spatial points $\mathbf{x}$ and $\mathbf{y}$. In this work, we use the following Gaussian kernel function [14]:

$$
\phi(\mathbf{x})=\frac{1}{\sigma \sqrt{\pi}} \exp \left(-\frac{\mathbf{x} \cdot \mathbf{x}}{\sigma^{2}}\right)
$$

\subsection{Network model}

Here, we describe a nonlocal network mathematical model corresponding to the nonlocal continuous model (2) and accounting for the brain data. The brain connectome can be modelled by a coarse-grain model of the continuous system. It is a weighted graph $\mathcal{G}$ with $V$ nodes and $E$ edges defined in a domain $\Omega$. Specifically, it can be considered as weights of the graph $\mathcal{G}$ is represented by the adjacency matrix $\mathbf{W}$ and helps to construct 
the graph of the Laplacian. For $i, j=1,2,3, \ldots, V$, the elements of $\mathbf{W}$ are

$$
W_{i j}=\frac{n_{i j}}{l_{i j}^{2}}
$$

where $n_{i j}$ is the mean fiber number and $l_{i j}^{2}$ is the mean length squared between the nodes $i$ and $j$. We define the graph of the Laplacian $\mathbf{L}$ as

$$
L_{i j}=\rho\left(D_{i i}-W_{i j}\right), \quad i, j=1,2,3, \ldots, V,
$$

where $\rho$ is the diffusion coefficient and $D_{i i}=\sum_{j=1}^{V} W_{i j}$ is the elements of the diagonal weighted degree matrix.

Likewise, similar to the nonlocal continuous PDE model (2), we define the nonlocal conversion for the network model for each of the nodes $j=1,2,3, \ldots, V$. For a fixed node $j$, we first find all the nodes $V_{j, 1}$ directly connected to the node $j$. Similarly, we find the immediate connecting nodes $V_{j, 2}$ connected with the nodes $V_{j, 1}$ and so on. In this way, we find all the sets of nodes $V_{j, 1}, V_{j, 2}, \ldots, V_{j, m_{j}}$ connected with the node $j$. Now, we sort all the nodes $V_{j, 1}$ according to minimum distances from the node $j$ and labelled as $V_{j, 1}^{s}$. Similarly, we sort all the set of nodes $V_{j, 2}, V_{j, 3}, \ldots, V_{j, m_{j}}$ according to shortest distances from the node $j$ through the edge

$n_{j}$ is the total numl $k_{1}, k_{2}, \ldots, k_{n_{j}}$ are the node $j$ i.e., $k_{1}=j$.
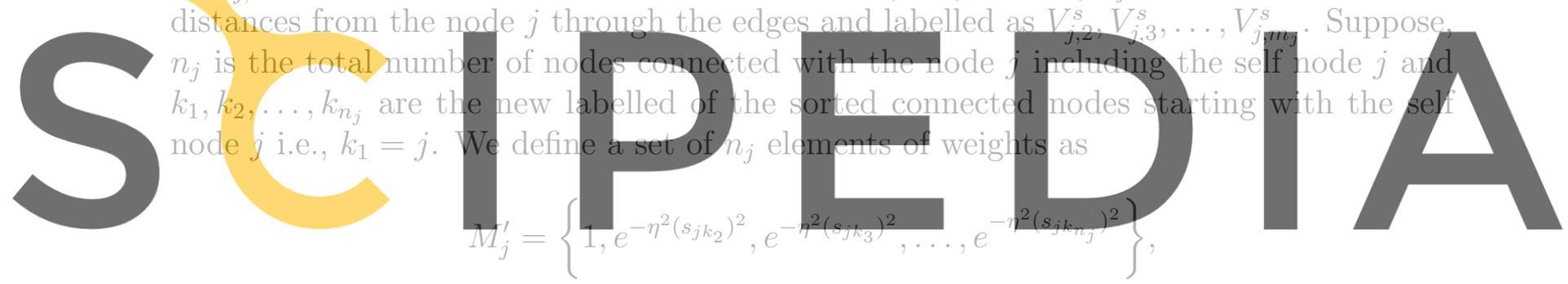

Register for free at https//www.scipedia.com to download the version without the watermark where $s_{j k_{i}}$ denotes the shortest distance from the node $k_{i}$ to the node $j$ along the edges and $\eta=1 / \sigma$.

We normalize $M_{j}^{\prime}$ as $M_{j}=M_{j}^{\prime} /\left|M_{j}^{\prime}\right|$, where

$$
\left|M_{j}^{\prime}\right|=\left(1+e^{-\eta^{2}\left(s_{j k_{2}}\right)^{2}}+e^{-\eta^{2}\left(s_{j k_{3}}\right)^{2}}+\cdots+e^{-\eta^{2}\left(s_{j k_{j}}\right)^{2}}\right) .
$$

Now, we define a row vector $C_{j}$ of $V$ number of elements with non-zero elements being $M_{j}(1), M_{j}(2), \ldots, M_{j}\left(n_{j}\right)$ at the indices $k_{1}, k_{2}, \ldots, k_{n_{j}}$ respectively. Finally, we are ready to define the convolution at the node $j$ in the graph $\mathcal{G}$ as

$$
\Phi_{j} * \widetilde{u}_{j}=\sum_{n=1}^{V} C_{j}(n) \widetilde{u}_{n} .
$$

If the node $j$ is not connected with the other nodes, then $\Phi_{j} * \widetilde{u}_{j}=\widetilde{u}_{j}$. Similarly, we define the other convolutions. 
Taking into account all the factors (Laplacian and convolutions), we build a nonlocal network mathematical model on the graph $\mathcal{G}$. At the node $j$, let $\left(u_{j}, \widetilde{u}_{j}\right)$ be the concentrations of healthy and toxic $A \beta$ and $\left(v_{j}, \widetilde{v}_{j}\right)$ be the concentrations of healthy and toxic $\tau P$, respectively. Then for all the nodes $j=1,2,3, \ldots, V$, the network equations corresponding to the continuous model (2) is a system of first order differential equations and it is given by

$$
\begin{aligned}
& \frac{d u_{j}}{d t}=-\sum_{k=1}^{V} L_{j k} u_{k}+a_{0}-a_{1} u_{j}-\frac{a_{2} u_{j}}{1+e_{1} u_{j}} \Phi_{j} * \widetilde{u}_{j}, \\
& \frac{d \widetilde{u}_{j}}{d t}=-\sum_{k=1}^{V} L_{j k} \widetilde{u}_{k}-\widetilde{a}_{1} \widetilde{u}_{j}+a_{2} \widetilde{u}_{j} \Phi_{j} *\left(\frac{u_{j}}{1+e_{1} u_{j}}\right),
\end{aligned}
$$

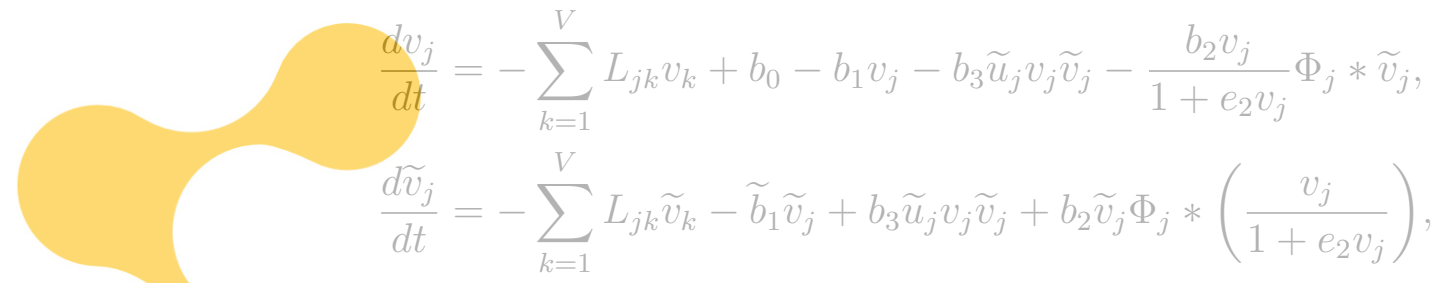

with non-negative init

2.4 Homogeneous
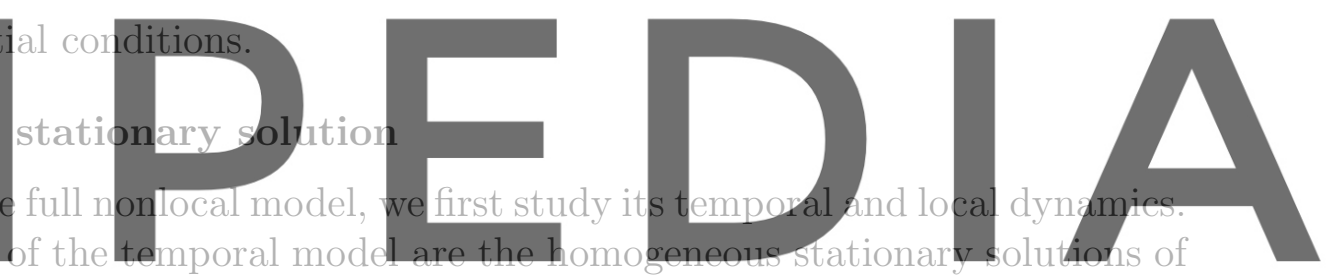

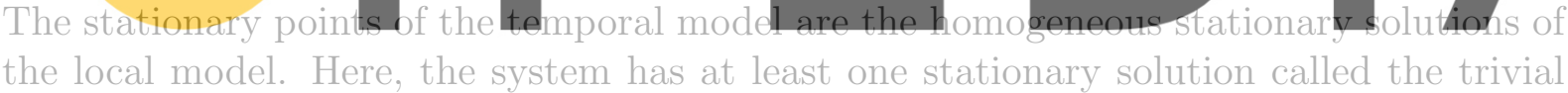

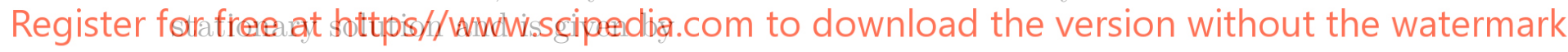

$$
\left(u_{1}, \widetilde{u}_{1}, v_{1}, \widetilde{v}_{1}\right)(\mathbf{x})=\left(\frac{a_{0}}{a_{1}}, 0, \frac{b_{0}}{b_{1}}, 0\right) .
$$

Depending on the parameter values, the system may have non-trivial stationary solutions. We list all of them in the below.

1. Healthy $A \beta$ - toxic $\tau P$ : The stationary solution corresponding to healthy $A \beta$ - toxic $\tau P$ is given by

$$
\left(u_{2}, \widetilde{u}_{2}, v_{2}, \widetilde{v}_{2}\right)(\mathbf{x})=\left(\frac{a_{0}}{a_{1}}, 0, \frac{\widetilde{b}_{1}}{b_{2}-e_{2} \widetilde{b}_{1}}, \frac{b_{0}\left(b_{2}-e_{2} \widetilde{b}_{1}\right)-b_{1} \widetilde{b}_{1}}{\widetilde{b}_{1}\left(b_{2}-e_{2} \widetilde{b}_{1}\right)}\right) .
$$

For the non-negativity of the stationary solution (6), we must have $b_{2}>e_{2} \widetilde{b}_{1}$ and $b_{0} / b_{1} \geq \widetilde{b}_{1} /\left(b_{2}-e_{2} \widetilde{b}_{1}\right)$. 
2. Toxic $A \beta$ - healthy $\tau P$ : The stationary solution corresponding to toxic $A \beta$ - healthy $\tau P$ is given by

$$
\left(u_{3}, \widetilde{u}_{3}, v_{3}, \widetilde{v}_{3}\right)(\mathbf{x})=\left(\frac{\widetilde{a}_{1}}{a_{2}-e_{1} \widetilde{a}_{1}}, \frac{a_{0}\left(a_{2}-e_{1} \widetilde{a}_{1}\right)-a_{1} \widetilde{a}_{1}}{\widetilde{a}_{1}\left(a_{2}-e_{1} \widetilde{a}_{1}\right)}, \frac{b_{0}}{b_{1}}, 0\right) .
$$

For the non-negativity of the stationary solution (7), we must have $a_{2}>e_{1} \widetilde{a}_{1}$ and $a_{0} / a_{1} \geq \widetilde{a}_{1} /\left(a_{2}-e_{1} \widetilde{a}_{1}\right)$.

3. Toxic $A \beta$ - toxic $\tau P$ : Suppose $\left(u_{4}, \widetilde{u}_{4}, v_{4}, \widetilde{v}_{4}\right)(\mathbf{x})$ is a stationary solution of the "toxic $A \beta$ - toxic $\tau P$ " type. Here, we have $u_{4}=u_{3}, \widetilde{u}_{4}=\widetilde{u}_{3}, \widetilde{v}_{4}=\left(b_{0}-b_{1} v_{4}\right) / \widetilde{b}_{1}$ and $v_{4}$ satisfy the quadratic equation

$$
b_{3} e_{2} \widetilde{u}_{4} v_{4}^{2}+\left(b_{3} \widetilde{u}_{4}-e_{2} \widetilde{b}_{1}+b_{2}\right) v_{4}-\widetilde{b}_{1}=0 .
$$

The equation (8) always has a real positive solution. For the uniqueness of $v_{4}$, we must have $b_{3} \widetilde{u}_{4}-e_{2} \widetilde{b}_{1}+b_{2} \geq 0$. Also, for the positivity of $\widetilde{v}_{4}$, we need $v_{4}<b_{0} / b_{1}$.

\section{RESULTS AND DISCUSSION}

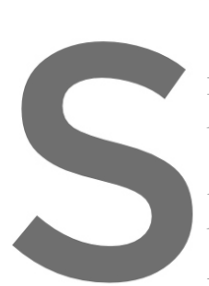

Here, we study two fundamental disease models depending tauopathy. For the A $\beta$ concentration, w these two tauopathics models in the brain
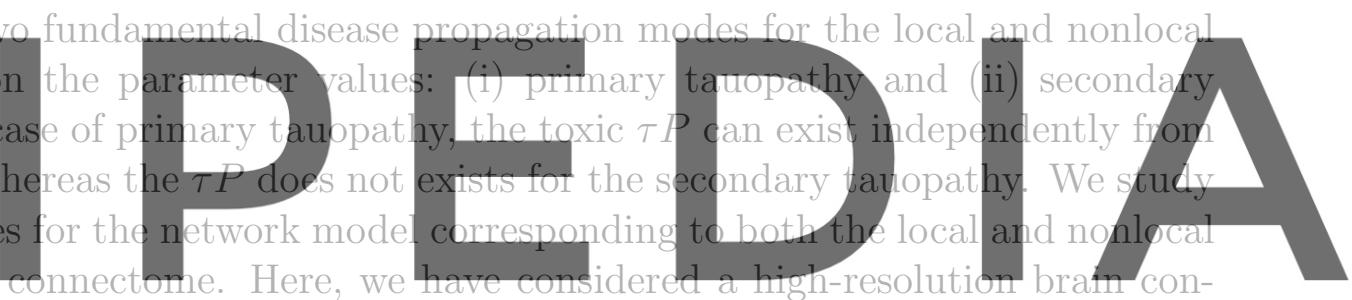
nectome structure consisting of 1,015 vertices and 70, 892 edges; the data source is freely

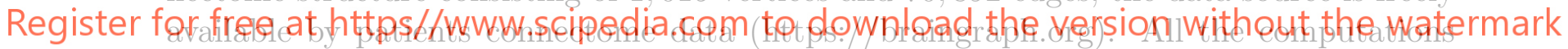
are done by using C programming language and Matlab.

Table 1: Fixed parameter values

\begin{tabular}{|c|c|}
\hline$A \beta$ system parameters & $\tau P$ system parameters \\
\hline$a_{0}=0.75$ & $b_{0}=0.5$ \\
\hline$a_{1}=1.0$ & $b_{1}=1.0$ \\
\hline$a_{2}=1.0$ & $b_{2}=1.0$ \\
\hline$\widetilde{a}_{1}=0.6$ & $\widetilde{b}_{1}=0.4$ \\
\hline
\end{tabular}

We apply the local and nonlocal versions of (4) to a computational case for Alzheimer's disease. In particular, we consider seeding sites for toxic $A \beta$ and toxic $\tau P$ proteins associated with Alzheimer's disease staging. Alzheimer's disease is a multiscale phenomenon. A uniform parameter value can be considered throughout all the regions in the brain 
or different values in different regions depending on the patient's disease progression. Initially, we investigate the primary and secondary tauopathies by considering simple uniform parameter values in all the regions. We choose the uniform parameters for both the tauopathies from Table 1.
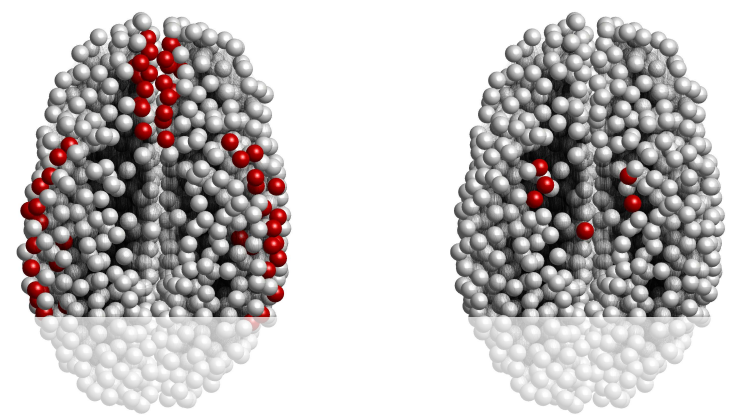

Figure 1: (Color online) Seeding sites for Alzheimer's disease: (left) toxic $A \beta$ and (right) toxic $\tau P$.

The seeding sites of toxic $A \beta$ protein associated with Alzheimer's disease are the temporobasal and fronton for the AD begins in follows from [19]. Seed respectively in Fig.

3.1 Primary tauopathy

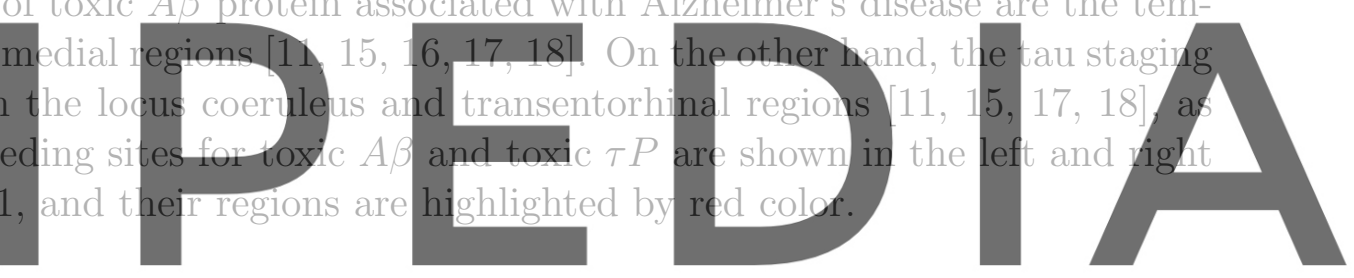

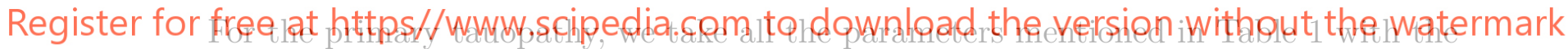

coupling parameter $b_{3}=1$. Here, all the stationary solutions (5)-(8) exist. Initially,

we set all the nodes in the brain connectome being healthy, but all are assumed to be susceptible. Therefore, the primary tauopathy state is the "healthy $A \beta$ - healthy $\tau P$ " state i.e., $\left(u_{1}, \widetilde{u}_{1}, v_{1}, \widetilde{v}_{1}\right)=(0.75,0,0.5,0)$. For the seeding sites of toxic $A \beta$, i.e., the temporobasal and frontomedial regions, initially we deviate $1 \%$ concentration of healthy concentration. Similarly, for the toxic $\tau P$, we perturb $1 \%$ of healthy concentration of $\tau P$ in the locus coeruleus and transentorhinal regions.

We first study the disease propagation by using the local network model. Brain-wide spatial averages of all the four protein populations at each time instance are shown in Fig. 2. The disease propagation is shown for four different values of $e_{1}$ and $e_{2}$. We also plot the propagation of toxic tau protein in the brain connectome for $e_{1}=e_{2}=0$ and $e_{1}=e_{2}=0.1$ in Fig. 3. For simplicity, we choose $e_{1}=e_{2}$. For higher values of $e_{1}$ and $e_{2}$, the local network model takes a long time to propagate the disease over the brain connectome. Therefore, the introduction of reaction time (described by the Holling type-II functional response) increases the invasion time window for propagating AD over 

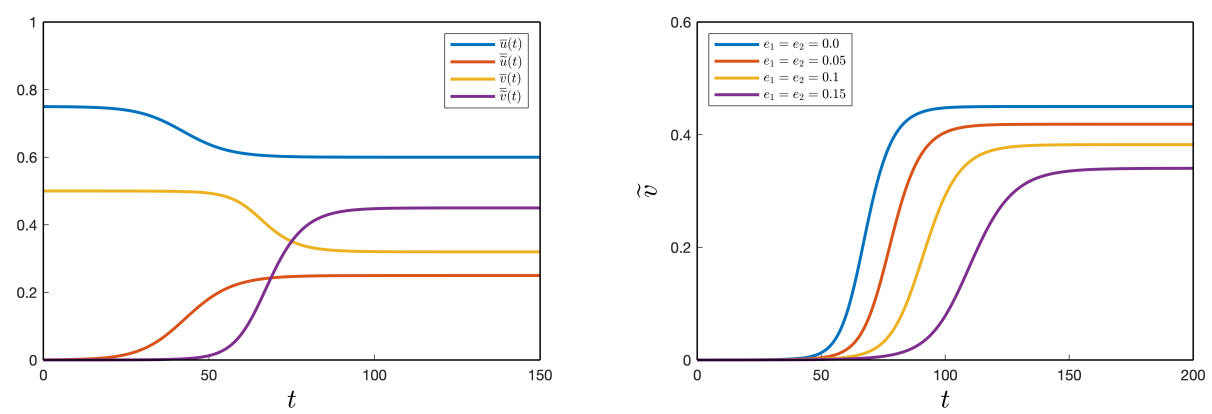

Figure 2: (Color online) Brain-wide spatial averages of the protein populations: (left) all four populations with $e_{1}=e_{2}=0$ and (right) toxic $-\tau P$ with different $e_{1}\left(=e_{2}\right)$.

the brain. It reduces the overall toxic level in the brain as well.
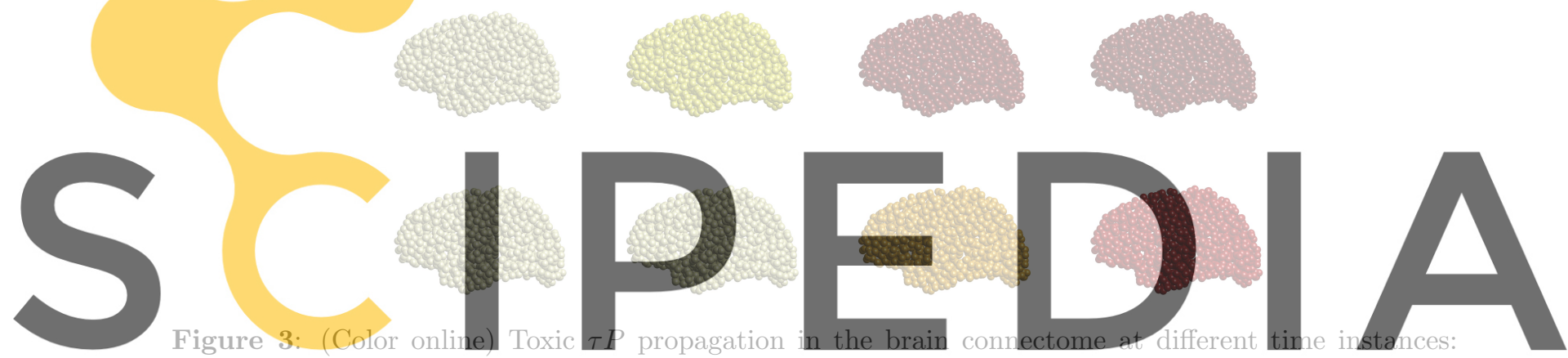

(left to right) $t=30,60,90$ and 120 ; Upper and lower panels are corresponding to $e_{1}=e_{2}=0$ and

Register for free at https//www.scipedia.com to download the version without the watermark

\subsection{Secondary tauopathy}

For the secondary tauopathy, we take the parameters from Table 1 except the parameter $b_{2}=0.75$ and we choose the coupling parameter $b_{3}=3$. In this case, the stationary solution (6) does not exist. We set all the nodes healthy but susceptible, and the initial condition is the same as for primary tauopathy [see Fig. 1]. The invaded concentrations of the four populations for the secondary tauopathy is the "toxic $A \beta$ - toxic $\tau P$ " state. The spreading patterns for the secondary tauopathy are similar to those of the primary tauopathy.

In both the tauopathies, the spreading patterns for the local and nonlocal models are the same. Their concentrations are also the same. This is due to the uniform parameter values all over the regions in the brain connectome. With the increase in the shortest distance between the connecting nodes, the weight-age of the nonlocal effect decreases exponentially. We have observed that the nonlocal interactions do not affect qualitatively 
the uniform parameter values over all the regions of the brain connectome. Now, we see the impact of nonlocal interactions on the results when the system has different parameter values in different regions of the brain connectome.

\subsection{Mixed tauopathies with non-uniform parameters}

Till now, we have considered some general features of the two tauopathies with synthetic and global constant parameter values. On the other hand, the positron emission tomography (PET) imaging studies of $A \beta$ and $\tau P$ radiotracer uptake provide us with additional insight. The distribution of PET- $\tau P$ SUVR intensities in Alzheimer's disease is biased towards the brain's temporal and parietal regions. These features are absent in the previous two tauopathies. Now, we study the model local and nonlocal effects with network system (4) along with the Alzheimer's disease patient data. A sample data is taken from the Alzheimer's Disease Neuroimaging Initiative (ADNI) database [11].
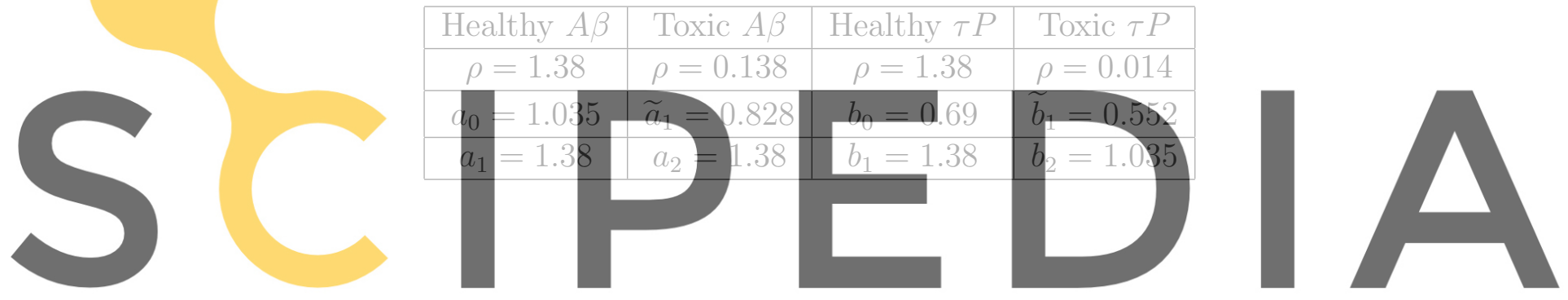

Table 3: Modified $b_{3}$ values

Register for free at https//www.scipedia.com to download the version without the watermark

\begin{tabular}{|c|c||c|c|}
\hline \multicolumn{5}{|c|}{ Brain region ID and modified $b_{3}$ value } \\
\hline Pars Opercularis & 7.452 & Rostral middle frontal gyrus & 6.707 \\
\hline Superior frontal gyrus & 7.452 & Caudal middle frontal gyrus & 7.452 \\
\hline Precentral gyrus & 5.589 & Postcentral gyrus & 3.726 \\
\hline Lateral orbitofrontal cortex & 6.486 & Medial orbitofrontal cortex & 6.486 \\
\hline Pars triangularis & $5.520 \mathrm{e}-6$ & Rostral anterior cingulate & $6.210 \mathrm{e}-6$ \\
\hline Posterior cingulate cortex & 3.45 & Inferior temporal cortex & 13.11 \\
\hline Middle temporal gyrus & 11.04 & Superior temporal sulcus & 8.97 \\
\hline Superior temporal gyrus & 8.28 & Superior parietal lobule & 12.42 \\
\hline Cuneus & 13.8 & Pericalcarine cortex & 13.8 \\
\hline Inferior parietal lobule & 11.73 & Lateral occipital sulcus & 15.18 \\
\hline Lingual gyrus & 13.8 & Fusiform gyrus & 7.59 \\
\hline Parahippocampal gyrus & 11.04 & Temporal pole & $1.104 \mathrm{e}-5$ \\
\hline
\end{tabular}


First, we set all regions in the connectome to a state of secondary tauopathy with the general synthetic parameters given in Table 2 with $b_{3}=4.14$. The coupling parameter $b_{3}$ is modified in several regions [see Table 3]. This modification is symmetric, i.e., $b_{3}$ has the same value for both the left and right hemispheres in the corresponding regions. We also modify $b_{2}$ and $b_{3}$ in some of the regions, and in this case, the system has a state of primary tauopathy [see Table 4]. After combining all the modified values, the system came up with a mixed state of tauopathy, i.e., a state of primary and secondary tauopathies exist simultaneously in different regions of the brain connectome.

Table 4: Modified $b_{2}$ and $b_{3}$ values

\begin{tabular}{|c|c|c|c|c|c|}
\hline Brain region & Entorhinal cortex & Pallidum & Locus cocrulcus & Putamen & Precuncus \\
\hline$b_{2}$ & 3.125 & 2.76 & 1.38 & 3.795 & 3.105 \\
\hline$b_{3}$ & $1.104 \mathrm{e}-5$ & 2.76 & 1.38 & 3.795 & 3.105 \\
\hline
\end{tabular}
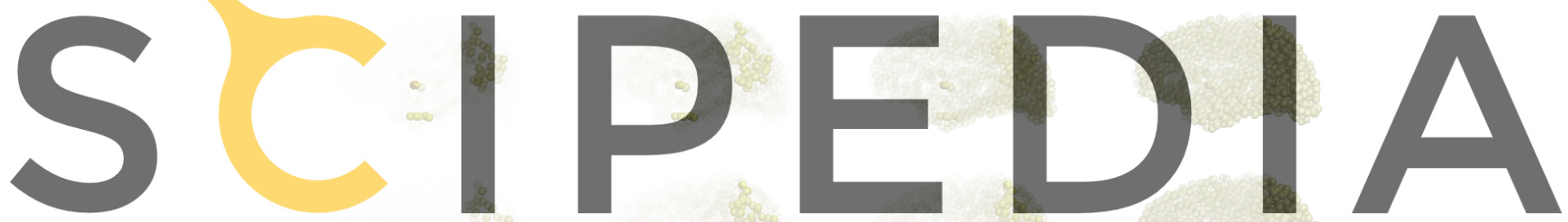

Register for free at https//www.scipedia.com to download the version without the watermark
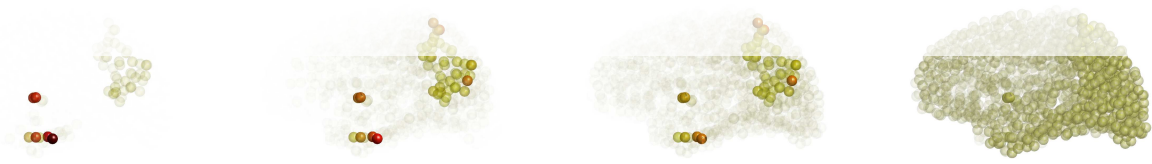

Figure 4: (Color online) Toxic $\tau P$ propagation in the brain connectome at different time instances: (left to right) $t=20,30,50$ and 100; Upper panel is corresponding to the local model; Middle and lower panels are corresponding to the nonlocal model with $\eta=0.5$ and $\eta=0.25$, respectively.

We solve the local and nonlocal variants network model (4) with the modified regional parameter values. The seeding sites of toxic $A \beta$ and $\tau P$ are mentioned earlier [see Fig. 1]. Toxic $\tau P$ propagation in the brain connectome for both the local and nonlocal versions are shown in Fig. 4 at $t=20,30,50$ and 100. The solution at $t=100$ is stationary, i.e., 
it remains the same for large values $t$. In this case, non-uniform concentrations of all four populations exist. This is true because the concentrations of toxic $A \beta$ and toxic $\tau P$ are not uniformly distributed in the real brain. We also see that the spreading patterns for both the local and nonlocal models are different for the mixed tauopathy.

\section{CONCLUSION}

We have considered a modified version of coupled heterodimer multiscale model for better understanding the dynamics of Alzheimer's disease. The network-based model corresponding to a PDE system is studied. For the first time, we have introduced nonlocal interactions into the considered network model. Two types of tauopathies (primary and secondary) are discussed separately, and their mixed tauopathy is also analysed. We have concluded that the spreading patterns and the invasion time windows for the local and nonlocal models for both the tauopathies are the same for the uniform parameters in all the regions of the brain connectome. However, the spreading patterns for the local and nonlocal models are different when mixed types of tauopathy are considered. Hence, the introduction of nonlocal interactions makes important qualitative changes in spreading all the four proteins over the brain.

\section{REFERENCES}

[1] Cornblath, E.J. et al. Defining and predicting transdiagnostic categories of neurodegenerative disease. Nature Biomedical Engineering (2020) 4:787-800.

[2] Vosoughi, A. et al. Mathematical models to shed light on amyloid-beta and tau protein dependent pathologies in Alzheimer's disease. Neuroscience (2020) 424:4557 .

[3] Walker, L.C. Lynn, D.G. and Chernoff, Y.O. A standard model of Alzheimer's disease?. Prion (2018) 12:261-265.

[4] Shaheen, H. Singh, S. and Melnik, R. A coupled neuronal-glial model for analyzing the effect of temperature on calcium-mediated exosomal dynamics. Submitted (2021).

[5] Reitz, C. Brayne, C. and Mayeux, R. Epidemiology of Alzheimer disease. Nat Rev Neurol. (2011) 7:137-152.

[6] Alzheimer Association. Alzheimer's disease facts and figures. Alzheimer's $\&$ Dementia (2017) 13:325-373.

[7] Querfurth, H.W. and LaFerla, FM. Alzheimer's disease. N Engl J Med (2010) 362:329-344.

[8] Kuznetsov, I.A. and Kuznetsov, A.V. How the formation of amyloid plaques and neurofibrillary tangles may be related: a mathematical modelling study. Proc. $R$. Soc. A (2018) 474:20170777. 
[9] Bressloff, P.C. and Newby, J.M. Stochastic models of intracellular transport. Rev. Mod. Phys (2013) 85:135.

[10] Soto, C. In Vivo Spreading of Tau Pathology. Neuron (2012) 73:621.

[11] Thompson, T.B. et al. Protein-protein interactions in neurodegenerative diseases: A conspiracy theory. PLoS Comput Biol (2020) 16:e1008267.

[12] Pal, S. and Melnik, R. Pathology dynamics in healthy-toxic protein interaction and the multiscale analysis of neurodegenerative diseases. Proc. of ICCS, Springer (2021) 13 pages.

[13] Banerjee, M. and Volpert, V. Prey-predator model with a nonlocal consumption of prey. Chaos (2016) 26:083120.

[14] Sherratt, J.A. Invasion Generates Periodic Traveling Waves (Wavetrains) in Predator -Prey Models with Nonlocal Dispersal. SIAM J. Appl. Math. (2016) 76:293-313.

[15] Jucker, M. and Walker L.C. Self-propagation of pathogenic protein aggregates in neurodegenerative diseases. Nature (2013) 501:45-51.

[16] Grothe, M.J. et al. In vivo staging of regional amyloid deposition. Neurology (2017) 89:2031-2038.

[17] Jucker, M. and Walker, L.C. Pathogenic protein seeding in Alzheimer disease and other neurodegenerative disorders. Annals of Neurology (2011) 70:532-540.

[18] Fornari, S. et al. Prion-like spreading of Alzheimer's disease within the brain's connectome. Interface $R$ Society (2019) 16:20190356.

[19] Braak, H. and Braak, E. Neuropathological stageing of Alzheimer-related changes. Acta Neuropathologica (1991) 82:239-259. 